\title{
PATRÓN DE CRECIMIENTO DURANTE LA PRIMERA GESTACIÓN DE VAQUILLONAS CRUZA CON PADRE BRAHMAN Y DIFERENTE GENOTIPO MATERNO
}

\section{GROWTH PATTERN DURING THE FIRST GESTATION OF HEIFERS CROSSES WITH BRAHMAN FATHER AND DIFFERENT MATERNAL GENOTYPE}

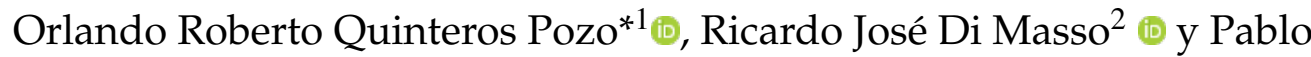 \\ Roberto Marini² ${ }^{2}$
}

\author{
${ }^{1}$ Centro Latinoamericano de Estudios de Problemáticas Lecheras (CLEPL), Universidad Estatal Amazónica, Calle Teniente Hu- \\ go Ortiz E45, Puyo, Ecuador \\ 2 Centro Latinoamericano de Estudios de Problemáticas Lecheras (CLEPL), Facultad de Ciencias Veterinarias, Universidad Na- \\ cional de Rosario, Maipú 1065, Santa Fe, Argentina \\ *Autor para correspondencia: oquinteros@uea.edu.ec
}

\begin{abstract}
Resumen
El objetivo del presente trabajo fue evaluar el patrón de crecimiento durante la primera gestación de vaquillonas cruza con padre Brahman y diferente genotipo materno en la Amazonía Ecuatoriana. Se utilizaron datos retrospectivos de 2304 pesos individuales de 48 vacas cruzas lecheras. Con ello, se evaluó el comportamiento dinámico del peso corporal de cuatro genotipos: Brahman x Gyr (Gyr) (n=12), Brahman x Brown Swiss (BS) (n=12), Brahman x Jersey (J) (n=12) y Brahman x Sahiwal (S) (n=12), pertenecientes al rodeo lechero del Centro de Investigación, Posgrado y Conservación de la Biodiversidad Amazónica (CIPCA) - Ecuador. Las diferencias entre genotipos en el peso corporal a la preñez y al primer parto no fueron estadísticamente significativas. Se observaron diferencias en la edad a la primera preñez correspondiendo la mayor precocidad a la cruza con madre Brown Swiss y la menor precocidad a las cruzas con Gyr y Sahiwal. Las hembras producto del cruzamiento con Jersey presentaron un comportamiento intermedio. Se observó que, durante su primera gestación, las vaquillonas F1 con padre Brahman y diferente genotipo materno presentan similar patrón de crecimiento en las condiciones limitantes de la Amazonía Ecuatoriana, respuesta interpretable en términos de interacción genotipo-ambiente negativa.
\end{abstract}

Palabras clave: edad al primer parto, aumento de peso, cruzamientos, interacción genotipo-ambiente, Amazonía. 


\begin{abstract}
The objective of the present work was to evaluate the growth pattern during the first gestation of heifers crossed with Brahman father and different maternal genotype in the Ecuadorian Amazon. Retrospective data of 2304 individual weights of 48 dairy cows were used. The dynamic behavior of the body weight of four genotypes was evaluated: Brahman x Gyr (Gyr) (n=12), Brahman x Brown Swiss (BS) ( $n=12)$, Brahman x Jersey (J) $(n=12)$ and Brahman $\times$ Sahiwal (S) $(n=12)$ belonging to the dairy herd of the Research Center, Postgraduate studies and Preservation of the Amazonia Biodiversity (CIPCA) - Ecuador. The differences between genotypes in body weight at pregnancy and at first birth were not statistically significant. Differences in age at the first pregnancy were observed, with the highest precocity at crossing with Brown Swiss mother and the lower precocity at crosses with Gyr and Sahiwal. The females produced by crossing with Jersey showed intermediate behavior. It was observed that, during their first gestation, F1 heifers with Brahman father and different maternal genotype have a similar growth pattern under the conditions of the Ecuadorian Amazon, response in terms of negative genotype-environment interaction.
\end{abstract}

Keywords: age at first calving, weight gain, crosses, genotype-environment interaction, Amazonia.

Forma sugerida de citar: Jiménez Ruíz, E. R., Fonseca González, W. y Pazmiño Pesantez, L. (2019). Patrón de crecimiento durante la primera gestación de vaquillonas cruza con padre Brahman y diferente genotipo materno. La Granja: Revista de Ciencias de la Vida. Vol. 30(2):40-47. http://doi.org/10.17163/lgr.n30.2019.04.

IDs Orcid:

Orlando Roberto Quinteros Pozo: https:/ / orcid.org/0000-0002-3808-257X

Ricardo José Di Masso: https:/ /orcid.org/0000-0003-4873-5156

Pablo Roberto Marini: https:/ / orcid.org/0000-0003-0826-0387

La Granja: Revista de Ciencias de la Vida 30(2) 2019:40-47.

(C)2019, Universidad Politécnica Salesiana, Ecuador. 


\section{Introducción}

Calderón y col., (1993) y Abeygunawardena y Dematawewa, (2004) informaron que las hembras provenientes de cruzamientos entre razas cebuinas y europeas, inician la pubertad a una edad más temprana que las razas puras en condiciones de trópico húmedo, con valores de 15 y 19 meses, respectivamente. La variabilidad genética existente tanto dentro como entre razas se traduce en diferencias en la edad y el peso corporal a la presentación del primer, afectando posteriores a eventos de carácter reproductivo que terminan siendo determinantes en la aptitud productiva global (Navarrete, 1995; Nogueira, 2004). Por esta razón, es importante contar con grupos raciales adaptados a estas condiciones particulares, que sean capaces de superar las limitantes productivas que presentan las razas de Bos taurus en estas regiones. En este sentido, se ha observado que las vacas producto de cruzamientos Brahman x Bos taurus presentan mayor fertilidad, mayor producción de leche y mayor longevidad que aquellas de genotipo Bos taurus puro (Grajales, Hernández y Prieto, 2006; Zambrano-Sepúlveda y col., 2014). Sin embargo, al aumentar la proporción de genes de origen índico, las hembras tienden a retrasar la edad a la pubertad y ello impacta negativamente en su comportamiento reproductivo posterior. En este sentido, se ha constatado (Rocha y Lobato, 2002; Zambrano y Contreras, 2014) la incapacidad de las vacas cruza Brahman x Bos taurus para producir una hembra de reemplazo que mantenga sus mismas características de productividad como consecuencia de la pérdida de la heterosis con respecto a la expresada por la F1.

El registro del peso vivo de los bovinos es una estrategia que permite monitorear el comportamiento en su entorno natural. Esta información se utiliza con diversos propósitos, entre los cuales cabe mencionar la determinación del nivel de alimentación adecuado y el estado nutricional de los animales, el control de la tasa de crecimiento y las respuestas a la selección genética (Marulanda, 1996; Lesosky y col., 2013; Lukuyu y col., 2016). En resumen, la evaluación del peso vivo de los animales en los sistemas de producción ganadera resulta fundamental si se desean implementar prácticas adecuadas de manejo de los pastizales tendientes al logro de una producción sustentable en la Amazonía. En este contexto, el objetivo del presente trabajo fue evaluar el patrón de crecimiento durante la primera gestación de vaquillonas cruza con padre Brahman y diferente genotipo materno en la Amazonía Ecuatoriana.

\section{Materiales y métodos}

Se utilizaron datos retrospectivos de 2304 registros individuales del peso corporal de 48 vacas cruzas lecheras pertenecientes a cuatro genotipos: Brahman x Gyr (Gyr) $(n=12)$, Brahman x Brown Swiss (BS) $(n=12)$, Brahman $x$ Jersey (J) $(n=12)$ y Brahman $x$ Sahiwal $(S)(n=12)$ pertenecientes al rodeo lechero del Centro de Investigación, Posgrado y Conservación de la Biodiversidad Amazónica (CIPCA), ubicado en el cantón Carlos Julio Arosemena Tola de la provincia de Napo (Ecuador), en el kilómetro 44 vía Puyo-Tena (coordenadas: S 01 14.325; O $77^{\circ} 53.134$ ) y dispone de una superficie de 42 ha de pastos destinada a la recría. Las vaquillonas evaluadas provenían del mismo establecimiento y habían sido criadas bajo las mismas condiciones ambientales, nutricionales y de manejo e ingresaron al CIPCA con 15-17 meses de edad y pesos corporales (promedio \pm EE) de $204 \pm 7,7 \mathrm{~kg}(\mathrm{G}) ; 276 \pm 11,0$ $\mathrm{kg}$ (BS); $204 \pm 8,7 \mathrm{~kg}$ (J) y $186 \pm 6,0 \mathrm{~kg}$ (S). A los fines de evaluar el comportamiento dinámico del peso corporal, todos los animales se pesaron cada 30 días en forma individual, entre los meses de diciembre de 2012 y diciembre de 2016. La alimentación del hato bovino en estudio fue de pastoreo libre, con pastizales en base de Brachiaria decumbens (17 $585 \mathrm{~kg}$ MS/ha/año, Proteína: 10,6\% Fósforo: 0,18\%; DIV: 44,4\%), Brachiaria brizantha (26 $970 \mathrm{~kg}$ MS/ha/año; Proteína: 10,1\%; Fósforo: 0,18\%; DIV: 44,1\%), Arachis pintoi (6 $212 \mathrm{~kg} \mathrm{MS} / \mathrm{ha} / \mathrm{año}$; Proteína: 19,4\%; Fósforo: 0,21\%; DIV: 59,2\%), Desmodium ovalifolium (5 $890 \mathrm{~kg}$ MS/ha/año; Proteína: 16,3\%; Fósforo: 0,16\%; DIV: 39,6\%) y Stylosanthes guianensis (15 $237 \mathrm{~kg} \mathrm{MS/ha/año;} \mathrm{Proteína:} \mathrm{21,4 \% ;}$ Fósforo: 0,4\%; DIV: 48,7\%) (Leonard, 2015).

El manejo sanitario aplicado fue el habitualmente empleado para el rodeo bovino del CIPCA, el cual incluye desparasitaciones, baños contra garrapatas y moscas, vacunaciones para fiebre aftosa, rabia bovina y estomatitis vesicular y la aplicación inyectable de vitaminas y minerales. Se registró en forma individual el peso corporal $(\mathrm{kg})$ a la primera preñez, la edad (días) a la primera preñez y el peso corporal $(\mathrm{kg})$ a la primera parición. La normalidad de la 
distribución de cada una de dichas variables se evaluó con el test ómnibus de D'Agostino y Pearson (D'Agostino y Pearson, 1973), y la homogeneidad de sus variancias con la prueba de Brown-Forsythe (Brown y Forsythe, 1974). Para cada genotipo se calculó el peso corporal promedio a intervalos mensuales durante la primera gestación. Los datos promedio se graficaron en función de los meses de gestación identificándose en todos los casos un comportamiento asimilable a un modelo lineal, el que se constató con un test de rachas o ciclos. El efecto del grupo genético sobre los valores de los estimadores de los parámetros de la función lineal se evaluó con un análisis de la covariancia.

\section{Resultados}

La Tabla 1 muestra el efecto del grupo genético sobre las tres variables respuesta. Todas ellas mostraron distribución normal y variancias homogéneas. Las diferencias observadas entre genotipos en el peso corporal a la preñez y al primer parto no fueron estadísticamente significativas. Se observaron diferencias en la edad a la primera preñez, correspondiendo la mayor precocidad a la cruza con madre Brown Swiss y la menor precocidad a las cruzas con Gyr y Sahiwal. Las hembras producto del cruzamiento con Jersey presentaron un comportamiento intermedio.

Tabla 1. Edad y peso corporal a la primera preñez en cuatro grupos de vaquillonas F1 con padre Brahman y diferente genotipo materno.

\begin{tabular}{cccccc}
\hline \multirow{2}{*}{ Variable } & \multicolumn{4}{c}{ Genotipo materno } & \multirow{2}{*}{ Contrastes } \\
\cline { 2 - 5 } & Gyr & Brown Swiss & Jersey & Sahiwal & \\
\hline Edad (días) & $1148^{c} \pm 57,7$ & $852^{a, b} \pm 38,3$ & $994^{b, c} \pm 49,5$ & $1135^{c} \pm 54,4$ & $\mathrm{~F}=7,619 \mathrm{p}=0,0003$ \\
\hline Peso preñez $(\mathrm{kg})$ & $319 \pm 17,3$ & $350 \pm 9,5$ & $308 \pm 13,6$ & $324 \pm 8,6$ & $\mathrm{~F}=1,239 \mathrm{p}=0,307$ \\
\hline Peso parto $(\mathrm{kg})$ & $392 \pm 15,8$ & $414 \pm 18,8$ & $399 \pm 14,0$ & $399 \pm 11,0$ & $\mathrm{~F}=1,239 \mathrm{p}=0,307$ \\
\hline
\end{tabular}

Todos los valores corresponden a la media aritmética \pm error estándar.

Tamaño muestral: $\mathrm{n}=12$ individuos por grupo.

a, b, c Valores con diferente letra difieren al menos al 0,05

$\mathrm{Al}$ ajustar los datos de peso corporal promedio vs meses de gestación se constató una desviación no significativa de la linealidad para los cuatro grupos genéticos [madre Gyr $(p=0,833)$, madre Brown Swiss $(p=0,283)$, madre Jersey $(p=0,881)$ y madre Sahiwal $(p=0,405)]$. Los valores del coeficiente de determinación lineal $\left(R^{2}\right)$ y las variancias residuales
$\left(S_{y x}\right)$ pusieron de manifiesto una adecuada bondad de los ajustes [madre Gyr $\left(R^{2}=0,773 ; S_{y x}=9,88\right)$, madre Brown Swiss $\left(R^{2}=0,958 ; S_{y x}=6,00\right)$, madre Jersey $\left(R^{2}=0,967 ; S_{y x}=5,54\right)$ y madre Sahiwal $\left(R^{2}=\right.$ 0,$\left.\left.975 ; S_{y x}=3,85\right)\right]$. Las ecuaciones de regresión correspondientes a los diferentes grupos se presentanen la Tabla 2.

Tabla 2. Regresión de los diferentes grupos de madres.

\begin{tabular}{cc}
\hline Madre Gyr & $\mathrm{Y}=5,676^{*} \mathrm{X}+321,2$ \\
\hline Madre Pardo suizo & $\mathrm{Y}=8,884 * \mathrm{X}+342,5$ \\
\hline Madre Jersey & $\mathrm{Y}=9,347 * \mathrm{X}+307,5$ \\
\hline Madre Sahiwal & $\mathrm{Y}=7,488^{*} \mathrm{X}+321,7$ \\
\hline
\end{tabular}

Los valores de la ordenada al origen (estimadores del peso corporal al inicio de la preñez) no mos- traron diferencias $(p>0,05)$ con los valores observados: 
Tabla 3. Ordenadas de la regresión de los diferentes grupos de madres.

\begin{tabular}{ccc}
\hline Genotipo & Ordenada & Peso registrado \\
\hline Madre Gyr & $\mathrm{a}=321,2 \mathrm{Kg}$ & $319 \mathrm{Kg}$ \\
\hline Madre Pardo suizo & $\mathrm{a}=342,5 \mathrm{Kg}$ & $350 \mathrm{Kg}$ \\
\hline Madre Jersey & $\mathrm{a}=307,5 \mathrm{Kg}$ & $308 \mathrm{Kg}$ \\
\hline Madre Sahiwal & $\mathrm{a}=321,7 \mathrm{Kg}$ & $324 \mathrm{Kg}$ \\
\hline
\end{tabular}

Tabla 4. Pendientes de la regresión de los diferentes grupos de madres.

\begin{tabular}{ccc}
\hline Genotipo & \multicolumn{1}{c}{ Regresión } & Significancia \\
\hline Madre Gyr & $\mathrm{b} \pm S_{b}=5,676 \pm 1,088 \mathrm{~kg} / \mathrm{mes}$ & $\mathrm{F}=27,2 \mathrm{p}=0,0008$ \\
\hline Madre Pardo suizo & $\mathrm{b} \pm S_{b}=8,884 \pm 0,661 \mathrm{~kg} / \mathrm{mes}$ & $\mathrm{F}=181 \mathrm{p}<0,0001$ \\
\hline Madre Jersey & $\mathrm{b} \pm S_{b}=9,347 \pm 0,610 \mathrm{~kg} / \mathrm{mes}$ & $\mathrm{F}=235 \mathrm{p}<0,0001$ \\
\hline Madre Sahiwal & $\mathrm{b} \pm S_{b}=7,488 \pm 0,423 \mathrm{~kg} / \mathrm{mes}$ & $\mathrm{F}=313 \mathrm{p}<0,0001$ \\
\hline
\end{tabular}

Todas las pendientes fueron estadísticamente diferentes de 0 . El análisis de la covariancia puso en evidencia una diferencia estadísticamente significativa en el valor de las pendientes $(F=5,018 ; p=$ $0,0058)$ de los diferentes grupos genéticos, lo que impidió comparar las diferencias en altura. Debido a que las pendientes estiman la tasa de ganancia diaria de peso a lo largo de la gestación, la diferencia observada entre las mismas puso en evidencia un comportamiento particular de las hembras pro- ducto del cruzamiento Brahman x Gyr que mostraron un menor aumento de peso en el período. Al excluir a dicho grupo del análisis, las diferencias entre las pendientes de los tres genotipos restantes fueron estadísticamente no significativas $(\mathrm{F}=2,845$; $\mathrm{p}$ $=0,078$ ) lo que permitió calcular una pendiente común a todas ellas (bc $=8,573 \mathrm{~kg} / \mathrm{mes}$ ) y comparar las alturas que resultan estadísticamente diferentes $(\mathrm{F}=99,4 ; \mathrm{p}<0,0001)$.

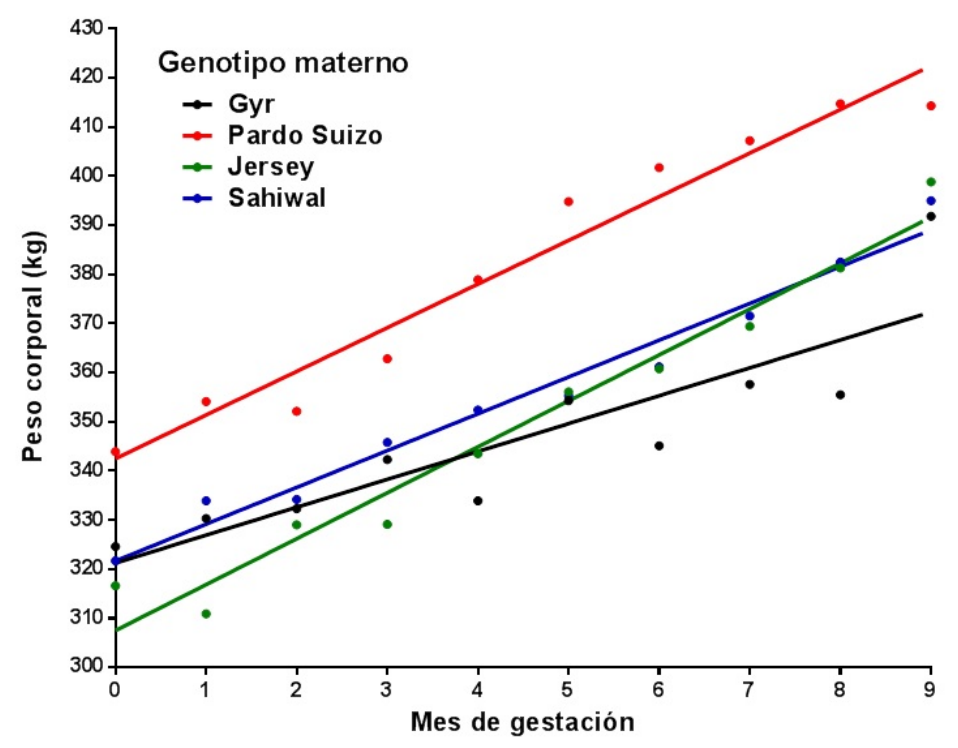

Figura 1. Patrón de crecimiento lineal durante la primera gestación de vacas cruza con genotipo paterno Brahman y diferente genotipo materno en condiciones de pastoreo en la Amazonia ecuatoriana. 


\section{Discusión}

En Ecuador, al igual que en otros países del trópico y el subtrópico de Latinoamérica, ha existido una fuerte tendencia a la sustitución de las razas locales por razas exóticas con el fin de incrementar los niveles de producción de leche. Estas razas, introducidas en la zona con fines de mejoramiento, provienen de regiones donde las condiciones climáticas y nutricionales son generalmente más favorables en comparación con las imperantes en la zona de destino, por lo que su rendimiento productivo en general se ve afectado negativamente. Ante esta evidencia, una estrategia de manejo genético a la cual han recurrido los productores locales es la utilización de cruzamientos entre razas cebuinas (Bos indicus) y razas europeas (Bos taurus), en un intento por minimizar los problemas de adaptación evidentes en estas últimas (Tewolde, 1993; Madalena, 2012).

Pese a sus diferencias de tamaño corporal, todos los genotipos evaluados en este trabajo mostraron similar comportamiento en términos de patrón de crecimiento durante su primera gestación. Este resultado coincide con evidencia previa, según la cual los aumentos de pesos mensuales durante la recría hasta el primer servicio de los cuatro genotipos lecheros cruza analizados fueron de 0,201 kg/día, acordes a la región en estudio. Ninguno de los cruzamientos lecheros se destacó por encima del otro, mostrando todos ellos una paridad en este aspecto (Quinteros y col., 2015).

El hecho que las vaquillonas en estudio no se diferencian en sus ganancias de peso durante la primera gestación, independientemente de su constitución genética, puede interpretarse en términos de una correlación genotipo-ambiente negativa. De acuerdo con ello, al mejor genotipo en términos de velocidad de crecimiento potencial -cruza con Brown Swiss- le corresponde comparativamente el peor ambiente. Esta afirmación se sustenta en el hecho que, si el peso a la preñez representó el $65 \%$ del peso adulto de cada genotipo materno, el peso al parto debería haber sido el $90 \%$ del peso adulto, con valores teóricos para Brahman x Gyr de $441 \mathrm{~kg}$, para Brahman x Brown Swiss de $538 \mathrm{~kg}$, para Brahman $x$ Jersey de $473 \mathrm{~kg}$ y para Brahman $x$ Sahiwal de $498 \mathrm{~kg}$. Esta predicción difiere de los resultados informados en este trabajo con pesos corporales observados menores a los esperados bajo dicha hipótesis (Tabla 1), y acordes con los que el ambiente limitante de la zona les permitió expresar. La elevada humedad relativa y las altas temperaturas características de la Amazonía ecuatoriana son factores que impactan negativamente sobre las vacas, y de acuerdo a lo planteado por Sánchez, (2010) y Arias, Mader y Escobar, (2008), los animales producirán en la medida que las condiciones del medio ambiente les permitan expresar su potencial productivo. La baja rentabilidad de las producciones refleja los valores resultantes de la evaluación de variables productivas y reproductivas, utilizadas como indicadores de su tasa o nivel de utilidad económica y adaptación de los animales, expresados bajo la forma de promedios de edad al primer parto, intervalo entre partos y presentación celo postparto y producción de leche (Morales, 2009; Motta y col., 2012; Quinteros-Pozo y Marini, 2017).

El grupo con genotipo materno Brown Swiss comienza la preñez a una edad más temprana (mayor precocidad), y si bien las diferencias no alcanzaron significado estadístico con mayor peso corporal, esa diferencia se mantiene a lo largo de toda la gestación. Probablemente una mejora en la calidad y en la cantidad de la dieta ofrecida podría aumentar la ganancia media diaria peso; sin embargo, la realidad indica que esta opción no es siempre posible, por lo que se deberían pensar alternativas haciendo uso de los elementos disponibles en la Amazonía. A este respecto, hay antecedentes que demuestran que valores de ganancia de peso comprendidos entre los 600 y 750 gramos por día serían óptimos para las futuras vacas lecheras, mientras que valores menores podrían ser causa de un retraso de la pubertad (Wathes y col., 2014; González-Stagnaro y col., 2007). Sin embargo, en general, se trata de trabajos que no han sido realizados en ambientes como el de la Amazonía, por lo que la transferencia de los resultados derivados de ellos no resulta siempre posible.

\section{Conclusión}

Los resultados permiten corroborar que las condiciones ambientales limitantes reinantes en la Amazonia Ecuatoriana, impiden la expresión de las diferentes potencialidades genéticas de las vaquillonas F1 con padre Brahman y diferente genotipo ma- 
terno durante su primera gestación, dando como resultado un similar patrón de crecimiento en todas ellas. Teniendo en cuenta que lo que se mide en los animales es un fenotipo que resulta de la acción de su composición genética particular en el medio ambiente en el que le toca expresarse, la elección de una cruza en particular estará determinada por la factibilidad de ofertar un ambiente nutricional más acorde con sus requerimientos. La presencia de una interacción genotipo-ambiente de signo negativo indica que carece de sentido generar por cruzamiento una población cuya potencialidad de crecimiento y su correlato a nivel reproductivo no puede expresarse por limitaciones atribuibles al ambiente.

\section{Referencias}

Abeygunawardena, H y CMB Dematawewa (2004). «Pre-pubertal and postpartum anestrus in tropical Zebu cattle». En: Animal Reproduction Science 82, 373-387. Online: https:/ / bit.ly/2YJcZbE.

Arias, RA, TL Mader y PC Escobar (2008). «Factores climáticos que afectan el desempeño productivo del ganado bovino de carne y leche». En: Archivos de medicina veterinaria 40.1, 7-22. Online: https://bit.ly/2yBzbtB.

Brown, Morton B y Alan B Forsythe (1974). «The small sample behavior of some statistics which test the equality of several means». En: Technometrics 16.1, 129-132. Online: https: / / bit.ly / 2Ko6ENK.

Calderón, RR y col. (1993). «Actividad ovárica en becerras Suizo Pardo y Cebú en clima tropical Af (c). I. Inicio de la pubertad y su correlación con la primera ovulación». En: Memorias Reunión Nacional de Investigación Pecuaria, INIFAP, Jalisco.

D’Agostino, Ralph y Egon S Pearson (1973). «Tests for departure from normality. Empirical results for the distributions of $b_{2}$ and $\sqrt{b_{1}} \gg$. En: Biometrika 60.3, 613-622. Online: https:/ / bit.ly/2YJcZbE.

González-Stagnaro, Carlos y col. (2007). «Primer servicio en novillas de doble propósito». En: Revista Científica 17.1, 39-46. Online: https: / / bit. ly/2YGerzx.

Grajales, H, A Hernández y E Prieto (2006). «Edad y peso a la pubertad y su relación con la eficiencia reproductiva de grupos raciales bovinos en el trópico colombiano». En: Livestock Research for Rural Development 18.10, 1-25. Online:https: / / bit.ly/2U7wVmS.
Leonard, I. (2015). «Recursos forrajeros autóctonos y promisorios para la ganadería en la provincia de Pastaza. Retos y posibilidades para una ganadería sostenible en la provincia de Pastaza de la Amazonia Ecuatoriana.» En: Universidad Estatal Amazónica. Cap. IV, págs. 46-69.

Lesosky, Maia y col. (2013). "A live weight-heart girth relationship for accurate dosing of east African shorthorn zebu cattle». En: Tropical Animal Health and Production 45.1, 311-316. Online:https: / / bit.ly /2Kgv9O9.

Lukuyu, Margaret N y col. (2016). «Use of body linear measurements to estimate liveweight of crossbred dairy cattle in smallholder farms in Kenya». En: SpringerPlus 5.1, 63. Online: https: / / bit.ly/2ZuwQwF.

Madalena, F. E. (2012). "Animal breeding and development-South American perspective». En: Journal of Animal Breeding and Genetics 129.3, 171-172. Online: https://bit.ly/2ODeFDP.

Marulanda, E (1996). «Evaluación de la edad al primer servicio y su incidencia en la productividad y reproducción de novillas Cebú». En: Revista El Cebú 287. Online:https:/ / bit.ly/2Kgvqk2.

Morales D., Pérez B. Botero R. (2009). Parámetros productivos y reproductivos de importancia económica en ganadería bovina tropical.

Motta, Delgado y col. (2012). «Productive and reproductive performance of F1 Gyr $\times$ Holstein cows in Colombian warm climate.» En: Veterinaria y Zootecnia 6.1, págs. 17-23.

Navarrete, M. (1995). «Análisis de productividad de diferentes cruzamientos en doble propósito. Experiencias en la costa norte colombiana». En: Estrategias de mejoramiento genético en la producción bovina tropical. Memorias seminario internacional. Medellín. Colombia, págs. 159-170.

Nogueira, G. P. (2004). «Puberty in south american bos indicus (Zebu) cattle». En: Animal reproduction science 82, 361-372. Online: https: / / bit.ly / 2yG9vMF.

Quinteros-Pozo, R. y P.R. Marini (2017). «Evaluación productiva y reproductiva de cuatro genotipos lecheros en pastoreo libre en la Amazonía ecuatoriana». En: Revista veterinaria 28.1, 9-13. Online: https://bit.ly/2yCt7ky.

Quinteros, O. R. y col. (2015). «Live Body Weight Evaluation of Four Genotypes of Dairy Heifers under Free Grazing Conditions in Ecuador». En: Asian Journal of Agriculture and Food Sciences 3.3, págs. $273-281$. 
Rocha, Marta Gomes da y José Fernando Piva Lobato (2002). «Avaliação do desempenho reprodutivo de novilhas de corte primíparas aos dois anos de idade». En: Revista brasileira de zootecnia. 31.3, 1388-1395. online: https://bit.ly/2Kgwnc6.

Sánchez, T.A. (2010). Análisis técnico económico del intervalo entre partos de bovinos. Inf. téc. Medellín, Colombia.: AFAGRO LTDA. Online: https:/ / bit. ly/2T5r0iX.

Tewolde, A. (1993). «El estado actual de los recursos genéticos animales en América Latina». En: Memoria Simposio sobre los Recursos Genéticos animales en América Latina, págs. 11-18.

Wathes, D. C. y col. (2014). "Heifer fertility and carry over consequences for life time production in dairy and beef cattle». En: Animal 8.1, 91-104. Online: https://bit.ly/2ZuCZJf.

Zambrano-Sepúlveda, Ramón Alfonso y col. (2014). «Evaluación de la vida productiva de un rebaño bovino mestizo doble propósito, utilizando análisis de supervivencia». En: Revista Científica 24.6, 535-542. Online: https:/ / bit.ly/2yzS3cG.

Zambrano, R. y R. Contreras (2014). «Descarte, vida productiva y selección en rebaños Doble Propósito». En: Logros Desafíos de la Ganadería Doble Propósito. C. González-Stagnaro, N. MadridBury, E. Soto-Belloso (Eds.). Fundación GIRARZ. Ediciones Astro Data SA Maracaibo, Venezuela. 16, págs. 157-164. 